\title{
Tunable Plasmon and Optical Properties of Chalcogenide Nanoplates Using Monochromated Electron Energy Loss Spectroscopy
}

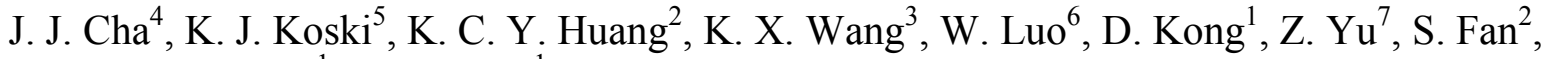
M. L. Brongersma ${ }^{1}$, and Y. Cui ${ }^{1}$

1. Department of Materials Science and Engineering, Stanford University, Stanford, USA

2. Department of Electrical Engineering, Stanford University, Stanford, USA

3. Department of Applied Physics, Stanford University, Stanford, USA

4. Department of Mechanical Engineering and Materials Science, Yale University, New Haven, USA

5. Department of Chemistry, Brown University, Providence, USA

6. Department of Physics and Astronomy, Shanghai Jiao Tong University, China

7. Department of Electrical and Computer Engineering, University of Wisconsin-Madison, Madison, USA

Following the success of graphene and boron nitride, two-dimensional (2D) layered metal chalcogenides are being intensely investigated for their electrical, chemical, and optical properties. For example, $\mathrm{Bi} 2 \mathrm{Se} 3$ and $\mathrm{Bi} 2 \mathrm{Te} 3$ are recently discovered topological insulators [1] while $\mathrm{MoS}_{2}$ is a promising catalyst for hydrogen evolution reaction (HER) [2, 3]. The layered crystal structure provides a unique opportunity to tune the materials properties by intercalation, a chemical process to insert guest species at the van der Waals gap [4, 5] (Figure 1A, schematic).

Dielectric properties of these metal chalcogenides are such that both dielectric photonic and surface plasmonic modes can be supported in ultrathin chalcogenide nanoplates. Using intercalation, we demonstrate that the optical and plasmonic properties of chalcogenide nanoplates can be tuned [6]. Monochromated electron energy loss spectroscopy (EELS) was used to directly image the propagation of the optical and plasmonic modes in chalcogenide nanoplates and to correlate changes in the optical and plasmonic modal properties as a function of intercalation. Monochromated EELS is the ideal technique [7] because it provides nanoscale resolution so that individual nanoplates can be tracked and studied before and after intercalation.

First, I will discuss the dispersion relation in momentum space, obtained from the real space EELS mapping of the optical and plasmon mode propagations (Figure $1 \mathrm{C}-1 \mathrm{H}$ ). Second, I will demonstrate the tuning of the optical and plasmonic modes by controlling the thickness of the nanoplates. Third, the optical and plasmonic modes will be tuned by changing the composition of the ternary chalcogenide nanoplates. Lastly and most importantly, I will show how the optical modes change in $\mathrm{Bi}_{2} \mathrm{Se}_{3}$ nanoplates with pyridine, nitrobenzene, and dodecylamine intercalation (Figure 2). The demonstration of tunable optical and plasmonic properties of 2D layered metal chalcogenides by intercalation points to a novel route to make atomic-scale metamaterials where the host chalcogenide and the intercalant exhibit two distinct dielectric properties. Anticipated functionality or applications of atomic-scale metamaterials may be quantum plasmonic effects or indefinite metamaterials. Monochromated EELS that gives nanoscale resolution is the ideal method to study these systems.

[1] H. Zhang et al, Nature Physics 5 (2009), p.438-442

[2] T. Jaramillo et al, Science 317 (2007), p.100-102 
[3] H. Wang et al, Proc. Natl. Acad. Sci. 110 (2013), p.19701-19706

[4] K. J. Koski et al, J. Am. Chem. Soc. 134 (2012), p.13773-13779

[5] K. J. Koski, J. J. Cha et al, J. Am. Chem. Soc. 134 (2012), p.7584-7587

[6] J. J. Cha et al, Nano Lett. 13 (2013), p.5913-5918

[7] J. J. Cha et al, Phys. Rev. B 81 (2010), 113102
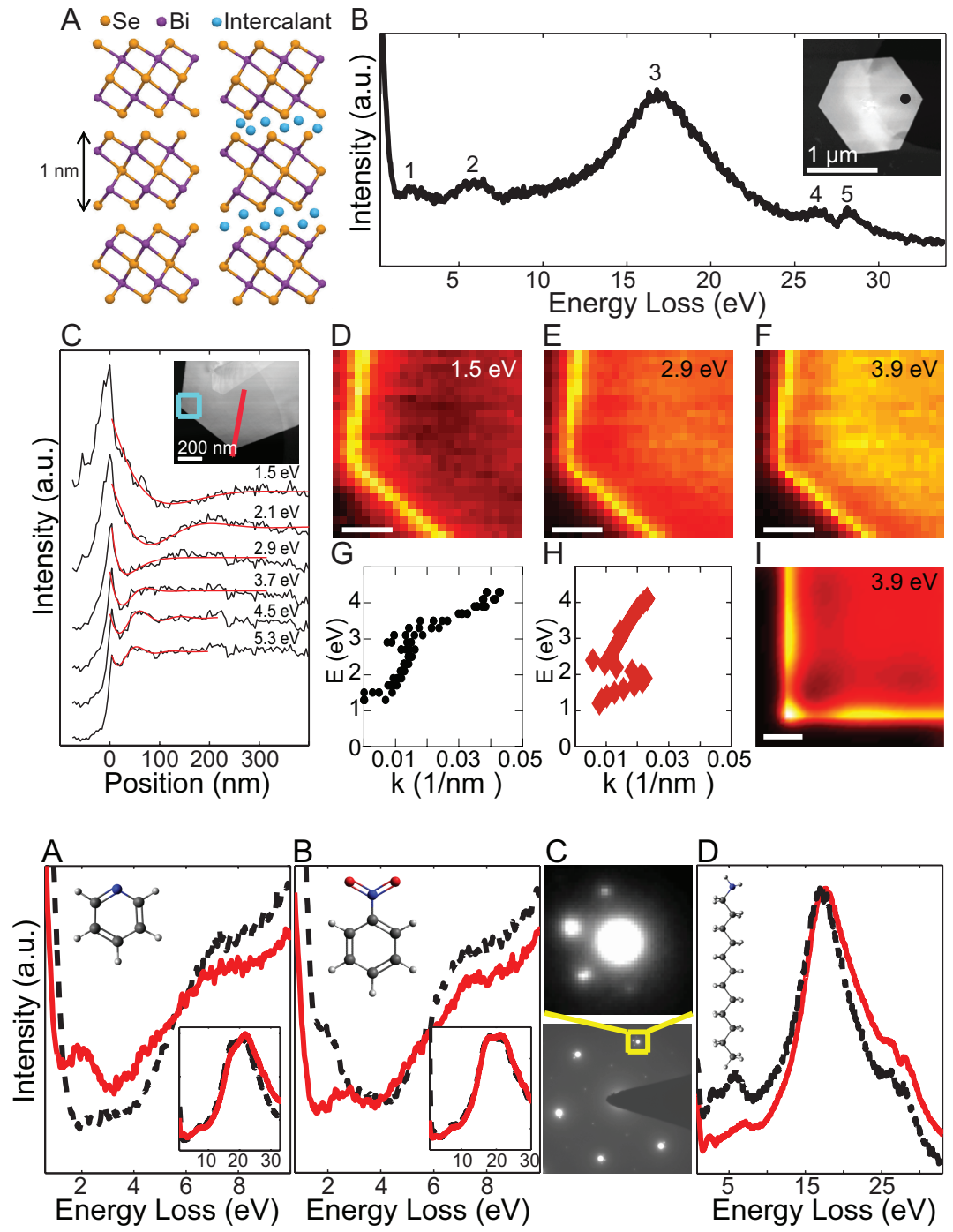

Figure 1. Photonic and plasmonic modes in $\mathrm{Bi}_{2} \mathrm{Se}_{3}$ nanoplates. (a) Crystal structure of $\mathrm{Bi}_{2} \mathrm{Se}_{3}$ before and after intercalation. (b) EELS from a $\mathrm{Bi}_{2} \mathrm{Se}_{3}$ nanoplate. Peak \#1 and 2 are the photonic and surface plasmon mode. (c) EELS intensity line traces at specific energies along the red line (inset image). (d-f) 2D maps of the photonic and plasmonic mode at specified energies $(1.5,2.9$, and 3.9 eV). (g) Experimentally obtained dispersion relation. (h) Calculated dispersion relation of a $10 \mathrm{~nm}$ thick $\mathrm{Bi}_{2} \mathrm{Se}_{3}$ slab. (i) Calculated 2D map of the surface plasmon of a $10 \mathrm{~nm}$ thick $\mathrm{Bi}_{2} \mathrm{Se}_{3}$ slab at $3.9 \mathrm{eV}$.

Figure 2. Optical mode changes before and after intercalation into $\mathrm{Bi}_{2} \mathrm{Se}_{3}$ nanoplates. EELS before (dotted black) and after (solid red) (a) pyridine, (b) nitrobenzene, and (d) dodecylamine intercalation. (c) Electron diffraction pattern of a dodecylamine-intercalated $\mathrm{Bi}_{2} \mathrm{Se}_{3}$ nanoribbon. Incommensurate charge density wave forms upon intercalation. 